\title{
Advances in the Treatment of Phantom Limb Pain
}

\author{
Dean H. Hommer · John P. McCallin • \\ Brandon J. Goff
}

Published online: 15 October 2014

(C) Springer Science+Business Media New York (Outside the USA) 2014

\begin{abstract}
Phantom limb pain (PLP) continues to place a significant emotional and physical burden on amputees and remains a challenge for those treating amputees. Despite advances in psychological, pharmacologic, and interventional therapies, treatment modalities and research results show promise, but there is no evidence to highly recommend any particular treatment. This review concludes that the best treatment approach is a measured and diligent trial of multiple modes of treatment. As researchers forge forward toward definitively establishing etiologies, focused treatment options may become available. Until then, PLP is an area that calls for intense research and which will continue to challenge the clinician caring for the amputee population.
\end{abstract}

Keywords Phantom limb pain · Neuropathic pain · Mirror therapy - Amputee rehabilitation .

Neuromodulation $\cdot$ Central sensitization

\section{Introduction}

The terms, phantom limb and phantom pain, were first used in the medical literature in the 1870 s by Silas Weir Mitchell, MD after observing the phenomenon in amputees

D. H. Hommer $(\bowtie) \cdot$ J. P. McCallin · B. J. Goff

San Antonio Military Medical Center, 3551 Roger Brooke Drive, JBSA-Fort Sam Houston, TX 78234, USA

e-mail: dean.h.hommer.mil@mail.mil

J. P. McCallin

e-mail: john.p.mccallin.mil@mail.mil

B. J. Goff

e-mail: brandon.j.goff.mil@mail.mil from the American Civil War. However, symptoms of a phantom limb may have been described 300 years earlier in the $1500 \mathrm{~s}[1,2 \cdot \cdot]$. Today, the prevalence of phantom limb pain (PLP) has been reported to be as high as $85 \%$ within 6 months following amputation [2••]. Phantom limb sensation occurs in almost all amputees and is neither painful nor distressing. This must be distinguished from PLP which is painful, distressing, and, sometimes, functionally disabling [3].

While the exact pathophysiology of PLP remains unknown, an understanding of potential etiologies continues to develop. Both central and peripheral changes have been observed to be potential contributors to PLP. Changes within the central nervous system, specifically neuroplasticity changes in the somatosensory cortical neurons, are seen in patients with PLP $[4 \bullet \bullet, 5]$. Changes in the neurochemical milieu are noted such as decreases in gammaamino-butyric acid (GABA)-ergic inhibition and glutamate-mediated long-term potentiation changes. There are also structural changes such as axonal sprouting [6]. Peripheral factors that may contribute include nociceptive input with the development of neuromas in the residual limb as well as, potentially, sympathetic contribution that may potentiate these sensations and pain [7]. Further, complicating the disease process is evidence that the structural and neurochemical changes are more extensive and pervasive when chronic pain precedes amputation, such as in a limb salvage patient [8]. Because of these multiple potential etiologies, it can be difficult to outline a standard treatment plan for PLP.

Whereas psychological therapies generally target conscious and subconscious pathways, pharmacologic therapies generally attempt to target a central pain mechanism behind PLP, though there are certainly peripheral effects from available mentioned medications. Interventional therapies 
are generally targeting potential peripheral causes of PLP but, with the increase in the use of neuromodulation, are now also targeting central mechanisms. This paper will outline current treatment options and the evidence to support each option.

\section{Pharmacologic Treatment}

Many classes of medications are used to treat PLP. Opioids, anticonvulsants, antidepressants, NMDA receptor antagonists, and calcitonins are among the medication classes used to treat pain. A Cochrane review in 2011 looked to determine the evidence for each of these medications classes in the treatment of PLP [9•]. A subsequent review in 2014 expanded on these medications for use in PLP [4••].

Studies involving different NMDA receptor antagonists to include memantine, dextromethorphan, and ketamine were included in the Cochrane review. Overall, the literature on NMDA receptor antagonists was equivocal. Looking at dextromethorphan and ketamine individually in smaller studies, each of these medications did show improvement in PLP in short-term studies. However, contrary to these studies, memantine did not show improvement in pain over a short time period [9•]. A more recent retrospective study looked at low-dose IV ketamine in combat-injured veterans, both with and without PLP, with pain refractory to multimodal oral and IV medication regimens in the acute setting. While there was a significant reduction in overall pain with the ketamine infusions, the effect was more pronounced in patients without PLP [10].

Anticonvulsants, specifically gabapentin, have been used extensively for neuropathic pain. The Cochrane review specifically reviewed two studies involving gabapentin. While these two studies did produce conflicting results, when the results of these studies were combined, there was a trend toward benefit from using gabapentin to treat PLP [9•]. A separate review in 2012 looking specifically at gabapentin for PLP again reviewed the same two articles as the Cochrane review but added a third study to the review. The conclusions were similar in this review, although the additional study reviewed did show a trend toward decreased opioid use when using gabapentin [11]. There is also some limited evidence for using topiramate for the treatment of PLP [4••].

Antidepressants are another class of medication used for neuropathic pain. The Cochrane review only found one article on the use of amitriptyline, a tricyclic antidepressant. There was no significant difference in the amitriptyline group compared to the placebo group for PLP [9•]. In multiple case series, the effectiveness of serotonin-norepinephrine reuptake inhibitors (SNRI), milnaciprin and duloxetine, as well as selective serotonin reuptake inhibitors (SSRI), has been established for PLP [4••, 12].

Calcitonin has been used widely in many disease states, and there has been some research on its use in treating PLP. Two studies have examined the use of calcitonin infusions for PLP; the first study did find that in acute PLP, calcitonin resulted in a significant reduction in pain compared to saline. In the other study looking at chronic PLP, calcitonin did not have a significant reduction in pain compared to ketamine, placebo or when used in combination with ketamine [9•]. Based on these two studies calcitonin may have more utility in the acute setting for PLP.

Opioids are mainstays for treatment of moderate to severe pain and PLP is no exception. However, only two studies were reviewed that examined morphine; one of these studies used injectable morphine, while the other used oral morphine. There was evidence, at least in the short term of less than 30 days, that morphine did result in a significant decrease in PLP [9•]. A recent case series looked at the use of the mixed opioid agonist-antagonist buprenorphine/naloxone for treatment of refractory PLP. In all four of these cases, there was a reduction of PLP. In all of these cases, the patient's PLP was refractory to multiple medications to include opioids, antidepressants, and anticonvulsants. Buprenorphine/naloxone may represent an alternative medication to consider in cases of refractory PLP [13].

All of the above medications have limited evidence to make clinical recommendations for the treatment of PLP and represent the need for more studies. However, because many of these medications have some evidence that trends toward benefit or evidence for their use in other types of chronic or neuropathic pain, it would be reasonable to consider them as part of a multimodal approach to treating PLP.

\section{Mirror Therapy}

Mirror therapy was first described in upper extremity amputees in 1996 [14]. Mirror therapy typically involves placing the amputated limb into a mirror box that reflects the intact limb and then putting the intact limb through a series of movements while observing these movements in the mirror to create the illusion of an intact limb [15] (Figure 1). After mirror therapy was first described in 1996, there has only been limited literature published on the use of mirror therapy in PLP in the form of multiple case reports and series [16, 17]. In 2007, two smaller randomized controlled trials were published that attempted to address this deficiency. One study looked at the effects of mirror therapy on phantom limb motion, phantom limb sensation, and PLP [18]. In this study, no differences were 


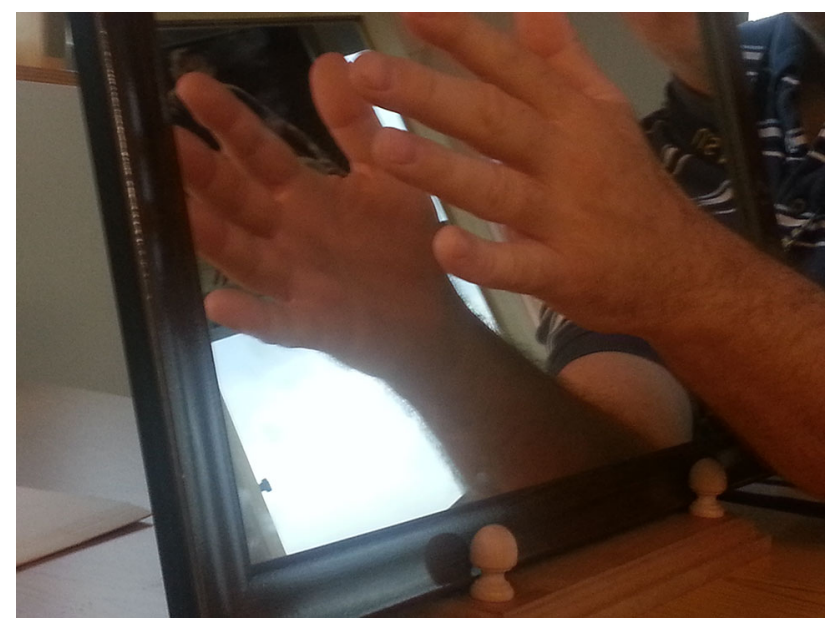

Fig. 1 Mirror Therapy: When the brain sees the mirror image of the left upper extremity, it interprets it as if the amputated right upper extremity is present, thus allowing the patient to "move" the right hand (Color figure online)

seen between the mirror therapy arm and the control arm. However, because this study was investigating multiple variables, some of the subjects did not report PLP. Another small randomized controlled study, investigating pain specifically, demonstrated a significant reduction of PLP with the use of mirror therapy [15].

Since these initial two randomized studies have been published, there has been limited additional research published on mirror therapy for PLP. All of the previous studies on mirror therapy involved therapist-supervised treatment with specific movement protocols. This increased resource utilization will likely increase cost and possibly decrease availability of this therapy [19]. Two studies have investigated ways to deliver mirror therapy in non-clinical settings specifically with home devices. This self-delivered home mirror therapy was first described in a case report where a patient purchased a long mirror to use at home. Use of mirror therapy at home resulted in complete resolution of his PLP [20]. A follow-up pilot study in 2012 did show a significant reduction in PLP with daily 25-min self-delivered home mirror therapy. However, the authors of this study acknowledged that the subjects with higher education had better outcomes than those with less education.

Given the relatively low cost of mirror therapy, a study which evaluated for any potential contraindications or side effects of mirror therapy was published in 2009. A small retrospective chart review from an acute inpatient rehabilitation facility was done. The majority of the patients reported some side effect to the therapy with over half of the patients reporting confusion and dizziness [21]. However, as this was a retrospective review and the subjects were in the acute rehabilitation phase, no conclusions about true side effects or absolute contraindications can be made.
While there is only limited evidence that mirror therapy results in a significant reduction of PLP, given the relatively low costs and few contraindications, mirror therapy should be considered as part of a treatment plan for PLP.

\section{Eye Movement Desensitization and Reprocessing}

While the power of the conscious to moderate PLP is powerful, the subconscious seems to play an equally powerful role in both eliciting and moderating PLP. In 2008, Schneider showed the role of psychology to have much promise in the treatment of PLP via a treatment known as eye movement desensitization and reprocessing (EMDR). In 2012, de Roos showed further success in treating PLP with EMDR $[22,23]$.

Patients with PLP report pain flashbacks, related to trauma-related cues, which are similar in quality and location to the original pain experience. They also report arousal and avoidance responses. Together, this points to the involvement of a somatosensory memory mechanism. These pain memories can be a significant factor in the maintenance of PLP, even after the injury leading to amputation has been effectively treated. Schneider and de Roos have continued to advance this treatment for chronic PLP. It is most promising in studies where it shows evidence for substantial long-term reduction of chronic PLP. Based on the available literature, where supported, EMDR should be considered as part of a comprehensive treatment program for PLP.

\section{Interventional Therapies}

Interventional treatments often target putative peripheral causes of PLP, but many address central pathologies as well. One of the pre-emptive techniques involves providing a continuous nerve blockade in the perioperative and postoperative period in an attempt to prevent the occurrence of PLP. There is conflicting evidence on the use of continuous epidural anesthesia in the prevention of PLP with some studies showing a lower incidence of PLP and others showing no difference in the incidence, although it does appear that perioperative pain control, in general, is important in attempting to prevent the development of PLP [24-26]. The literature on continuous perioperative and postoperative peripheral nerve block is similar to epidural anesthesia again with conflicting results in multiple small studies [24, 25, 27].

Outside of the perioperative and postoperative period, when PLP has developed, there have been several publications, primarily case reports and small pilot studies, on the use of peripheral nerve blocks or continuous nerve 
blockade in patients with refractory PLP [28-31]. While these results have been promising, the evidence is too limited to be able to provide a definitive recommendation and represents a need for further research.

Cryoneuroablation or cryoneurolysis is a technique that has been used for long-term pain control for many years by using the application of cold to a peripheral nerve, essentially causing Wallerian degeneration of the nerve. Again, the evidence is limited, but a recent retrospective case series reported on five patients who underwent cryoneuroablation, after a successful diagnostic nerve block with neurostimulation, resulting in some pain relief in all of the patients and 90-100\% pain relief in three of the patients [5]. Definitive recommendations cannot be made, but cryoneuroablation represents a promising potential treatment for PLP.

Neuromodulation in the form of spinal cord stimulation (SCS) or peripheral nerve stimulation has been used for many years in the treatment of many different chronic pain syndromes and post amputation pain, including PLP, is no exception. While the literature is variable in the long-term response, the majority of the patients studied had refractory PLP and did have some improvement, at least in the first year after implantation [32]. Peripheral nerve stimulation also is variable in the response but has some promising limited results in refractory PLP [33]. As technology and techniques for placement of these devices continue to evolve, improvement in pain control may continue to be seen in PLP with neuromodulation.

Botulinum toxin has been used for multiple different pain syndromes and has been suggested to have some inherent analgesic properties, in addition to its known function in providing a neuromuscular junction blockade. While there have been some case reports and series that showed promise for the treatment of PLP with botulinum toxin injection, a more recent small, randomized, doubleblinded study comparing Botulinum toxin to a lidocaine/ depomedrol mixture did not show significant reduction in PLP [4••, 34].

\section{Conclusion}

The treatment of PLP remains a perplexing and challenging problem in the amputee population. The exact etiology of PLP remains unknown, and there is a general lack of large studies or evidence to strongly support any of the treatments reviewed, however, these treatments, have shown varying degrees of benefit in some patients. While more research is needed, especially as technology continues to develop, this review underlines the importance of diligently designing a multimodal treatment plan for each patient consisting of trials of non-pharmacologic therapies, such as mirror therapy, pharmacologic therapies, and interventional therapies.

Disclaimer The view(s) expressed herein are those of the author(s) and do not reflect the official policy or position of Brooke Army Medical Center, the U.S. Army Medical Department, the U.S. Army Office of the Surgeon General, the Department of the Army and Department of Defense or the U.S. Government.

\section{Compliance with Ethics Guidelines}

Conflict of Interest DH Hommer, JP McCallin, and BJ Goff all declare no conflicts of interest.

Human and Animal Rights and Informed Consent This article does not contain any studies with human or animal subjects performed by any of the authors.

\section{References}

Papers of particular interest, published recently, have been highlighted as:

- Of importance

•• Of major importance

1. Aldington D, Small C, Edwards D, Ralph J, Woods P, Jagdish S, et al. A survey of post-amputation pains in serving military personnel. J R Army Med Corps. 2014;160(1):38-41.

2. $\bullet$ Hsu E, Cohen SP. Postamputation pain: epidemiology, mechanisms, and treatment. J pain res. 2013;6:121-36. An updated review of proposed mechanisms of PLP.

3. Le Feuvre P, Aldington D. Know pain know gain: proposing a treatment approach for phantom limb pain. J R Army Med Corps. 2014;160(1):16-21.

4. • McCormick Z, Chang-Chien G, Marshall B, Huang M, Harden RN. Phantom limb pain: a systematic neuroanatomical-based review of pharmacologic treatment. Pain Med (Malden, MA). 2014;15(2):292-305. Provides an updated review of all pharmacologic treatments of PLP.

5. Moesker AA, Karl HW, Trescot AM. Treatment of phantom limb pain by cryoneurolysis of the amputated nerve. Pain Pract. 2014;14(1):52-6.

6. Deer TR, Leong MS, Buvanendran A, Gordin V, Kim PS, Panchal SJ, et al. Comprehensive treatment of chronic pain by medical, interventional, and integrative approaches: The American Academy of Pain Medicine textbook on patient management. Springer; 2013.

7. Jaeger H, Maier C. Calcitonin in phantom limb pain: a doubleblind study. Pain. 1992;48(1):21-7.

8. Flor H. Maladaptive plasticity, memory for pain and phantom limb pain: review and suggestions for new therapies. Expert Rev Neurother. 2008;8(5):809-18.

9. - Alviar MJ, Hale T, Dungca M. Pharmacologic interventions for treating phantom limb pain. The Cochrane database of systematic reviews. 2011;(12):Cd006380. The most recent Cochrane review of pharmacologic treatments of PLP.

10. Polomano RC, Buckenmaier CC 3rd, Kwon KH, Hanlon AL, Rupprecht C, Goldberg C, et al. Effects of low-dose IV ketamine on peripheral and central pain from major limb injuries sustained in combat. Pain Med (Malden, MA). 2013;14(7):1088-100.

11. Abbass K. Efficacy of gabapentin for treatment of adults with phantom limb pain. Ann Pharmacother. 2012;46(12):1707-11. 
12. Nagoshi $Y$, Watanabe A, Inoue $S$, Kuroda $T$, Nakamura $M$, Matsumoto $\mathrm{Y}$, et al. Usefulness of milnacipran in treating phantom limb pain. Neuropsychiatric Dis Treat. 2012;8:549-53.

13. Licina L, Hamsher C, Lautenschager K, Dhanjal S, Williams N, Spevak C. Buprenorphine/Naloxone therapy for opioid refractory neuropathic pain following traumatic amputation: a case series. Mil Med. 2013;178(7):e858-61.

14. Ramachandran VS, Rogers-Ramachandran D. Synaesthesia in phantom limbs induced with mirrors. Proceedings Biological Sciences/The Royal Society. 1996;263(1369):377-86.

15. Chan BL, Witt R, Charrow AP, Magee A, Howard R, Pasquina $\mathrm{PF}$, et al. Mirror therapy for phantom limb pain. New Engl J Med. 2007;357(21):2206-7.

16. Moseley GL, Gallace A, Spence C. Is mirror therapy all it is cracked up to be? Current evidence and future directions. Pain. 2008;138(1):7-10.

17. Kim SY, Kim YY. Mirror therapy for phantom limb pain. Korean J Pain. 2012;25(4):272-4.

18. Brodie EE, Whyte A, Niven CA. Analgesia through the lookingglass? A randomized controlled trial investigating the effect of viewing a 'virtual' limb upon phantom limb pain, sensation and movement. Eur J Pain (London, England). 2007;11(4):428-36.

19. Darnall BD, Li H. Home-based self-delivered mirror therapy for phantom pain: a pilot study. J Rehabil Med. 2012;44(3):254-60.

20. Darnall BD. Self-delivered home-based mirror therapy for lower limb phantom pain. Am J Phys Med Rehabil. 2009;88(1):78-81.

21. Casale R, Damiani C, Rosati V. Mirror therapy in the rehabilitation of lower-limb amputation: are there any contraindications? Am J Phys Med Rehabil. 2009;88(10):837-42.

22. de Roos C, Veenstra AC, de Jongh A, den Hollander-Gijsman M, van der Wee NJ, Zitman FG, et al. Treatment of chronic phantom limb pain using a trauma-focused psychological approach. Pain Res Manag. 2010;15(2):65-71.

23. Schneider J, Hofmann A, Rost C, Shapiro F. EMDR in the treatment of chronic phantom limb pain. Pain Med (Malden, MA). 2008;9(1):76-82.

24. Becotte A, de Medicis E, Lapie V, Martin R, Gagnon V. Preoperative continuous sciatic nerve block for perioperative analgesia and for phantom limb prevention. J Clin Anesth. 2012;24(3):256-7.
25. Sahin SH, Colak A, Arar C, Tutunculer E, Sut N, Yilmaz B, et al. A retrospective trial comparing the effects of different anesthetic techniques on phantom pain after lower limb amputation. Curr Ther Res Clin Exp. 2011;72(3):127-37.

26. Karanikolas M, Aretha D, Tsolakis I, Monantera G, Kiekkas P, Papadoulas S, et al. Optimized perioperative analgesia reduces chronic phantom limb pain intensity, prevalence, and frequency: a prospective, randomized, clinical trial. Anesthesiology. 2011;114(5):1144-54.

27. Borghi B, D'Addabbo M, White PF, Gallerani P, Toccaceli L, Raffaeli W, et al. The use of prolonged peripheral neural blockade after lower extremity amputation: the effect on symptoms associated with phantom limb syndrome. Anesth Analg. 2010;111(5):1308-15.

28. Ilfeld BM, Moeller-Bertram T, Hanling SR, Tokarz K, Mariano ER, Loland VJ, et al. Treating intractable phantom limb pain with ambulatory continuous peripheral nerve blocks: a pilot study. Pain Med (Malden, MA). 2013;14(6):935-42.

29. Neil MJ. Brachial plexus block in phantom limb pain: interesting, but limited clinical benefit in chronic setting. Pain Med (Malden, MA). 2012;13(6):849.

30. Preissler S, Dietrich C, Meissner W, Huonker R, Hofmann GO, Miltner WH, et al. Brachial plexus block in phantom limb pain: a case report. Pain Med (Malden, MA). 2011;12(11):1649-54.

31. Preissler S, Meissner W, Weiss T. Brachial plexus block in phantom limb pain: not only interesting, but (sometimes) with clinical benefit. Pain Med (Malden, MA). 2012;13(6):850-1.

32. McAuley J, van Groningen R, Green C. Spinal cord stimulation for intractable pain following limb amputation. Neuromodulation. 2013;16(6):530-6 discussion 6.

33. Rauck RL, Cohen SP, Gilmore CA, North JM, Kapural L, Zang $\mathrm{RH}$, et al. Treatment of post-amputation pain with peripheral nerve stimulation. Neuromodulation. 2014;17(2):188-97.

34. Wu H, Sultana R, Taylor KB, Szabo A. A prospective randomized double-blinded pilot study to examine the effect of botulinum toxin type A injection versus Lidocaine/Depomedrol injection on residual and phantom limb pain: initial report. Clin J Pain. 2012;28(2):108-12. 